\title{
Oral hygiene practices and periodontal disease status assessment among diabetic patients from three selected public medical primary care clinics in Kuantan, Malaysia
}

Tin Myo Han $^{\mathrm{a}} \mid$ Razida Ismail ${ }^{\mathrm{b}} \mid$ Munirah Yaacob $^{\mathrm{a}} \mid$ Mohd Aznan Md Aris $^{\mathrm{c}}$ | Iskandar Firzada Osman ${ }^{\mathrm{d}}$ | Sorayah Sidek $^{\mathrm{e}}$ | Mahendran Thuraiappah ${ }^{f}$ | Fa'iza Abdullah ${ }^{\mathrm{c}}$ | Than Tun Sein ${ }^{\mathrm{g}}$ | Roslan Bin Saub ${ }^{\mathrm{h}}$

${ }^{a}$ Kulliyyah of Dentistry, International Islamic University Malaysia

${ }^{b}$ Periodontal Specialists' clinics, Klinik Kesihatan Paya Besar, Kuantan, Pahang, Malaysia

'Kulliyyah of Medicine, International Islamic University Malaysia

${ }^{d}$ Family Medicine Specialist, Klinik Kesihatan Jaya Gading, Kuantan, Pahang, Malaysia

'Periodontal Specialists' clinic, Klinik Kesihatan Presinct 18, Putrajaya

${ }^{f}$ Primary Care Department, Faculty of Medicine, MAHSA University, Kuala Lumpur, Malaysia

${ }^{g}$ Anthropology Department, Yangon University, Myanmar

${ }^{h}$ Faculty of Dentistry, University Malaya, Kuala Lumpur

Introduction: Evidences on the bilateral relationship between diabetes mellitus (DM) and periodontal diseases (PD) have been growing. Oral hygiene practice (OHP) is one of major determinants for PD. Thus, the aim of this study was to assess periodontal disease status and oral hygiene practices of DM-patients from public medical primary care clinics (PMPCCs). Methods: A medical-dental research team conducted an active PD-screening among 193 DMpatients using both self-reported questions (SRQs) and basic periodontal examination (BPE) by professionals at 3-PMPCCs in Kuantan in 2015. OHP was categorized into two groups; acceptable OHP (two/three-time tooth-brushing/day using with/without mouthwash/flossing) and need to improve OHP (one-time tooth brushing/day using with/without mouth-wash/flossing). $\mathrm{HbA} 1 \mathrm{C} \leq 6.5 \%$ was used as cut-off for glycaemic control achievement. A cross- analysis was done to infer the influences of demographic-background and OHP on PDstatus and relationship between PD- status and glycaemic control achievement. Results: Out of 193 DM-patients, $72.5 \%$ (140/193) were PD-screening positive in self-reporting while $54.9 \%$ $(106 / 193)$ had PD in professional screening. OHP of majority (86\%) were acceptable. Only $14 \%$ (27/193) achieved glycaemic-control status. Influence of demographic and OHP on PD-status ( by BPE) and relationship between PD and glycaemic control achievement did not found out. There were no age and race difference in OHP; however, acceptable OHP was significantly higher $(p<0.05)$ in female than male DM-patients $(94 \%$ vs $77.4 \%)$. Conclusions: High prevalence of PD indicated to promote oral health education/care among DM-patients from PMPCCs. In-detailed OHP/PD assessment and other influencing factors on glycaemic-control achievement should be considered to get more valid results in further study.

KEYWORDS: DM-Patients, Periodontal Disease screening, Oral Hygiene Practices, Medical Primary Care 